\title{
Semicontinuity Problems in the Calculus of Variations
}

\author{
EMILIo ACERBi \& Nicola Fusco
}

\author{
Communicated by J. SERRIN
}

\section{Introduction}

Let $f: \mathbb{R}^{\mathbf{3}} \rightarrow \mathbb{R}$ be a twice continuously differentiable function, and for $u \in W^{1,1}(a, b)$ set

$$
F(u ; a, b)=\int_{a}^{b} f\left(x, u(x), u^{\prime}(x)\right) d x .
$$

In a paper of ToneLLr [17] it is proved that the functional $F$ is lower semicontinuous (lsc) in the topology of $L^{\infty}(a, b)$ if and only if the function $f$ is convex in the last variable. Later, several authors generalized this result: among the many theorems obtained, in which $x$ is allowed to belong to $\mathbb{R}^{n}$ and considerably less regularity on $f$ is required, we recall particularly Theorem 12 of SERRIN [15], in which for the first time differentiability conditions on $f$ are dropped, and the following result due to MARCELlini \& SBORDONE [11]:

If $f: \mathbb{R}^{n} \times \mathbb{R} \times \mathbb{R}^{n} \rightarrow \mathbb{R}$ satisfies:

(i) $f$ is measurable in $x$, and continuous in $(s, \xi)$, and

(ii) $0 \leqq f(x, s, \xi) \leqq g(x,|s|,|\xi|)$,

where $g$ is increasing in $|s|$ and $|\xi|$, and is locally summable in $x$, then the functional

$$
F(u, \Omega)=\int_{\Omega} f(x, u(x), D u(x)) d x
$$

is sequentially weakly lower semicontinuous ${ }^{\star}$ on $W^{1, p}(\Omega)$, with $1 \leqq p \leqq+\infty$, if and only if $f$ is convex in $\xi$.

(See also Ekeland and TEMam [8] for the case in which $f$ does not depend on $u$ ).

$\star$ That is, $F(u, \Omega) \leqq \liminf F\left(u_{n}, \Omega\right)$ whenever $u_{n} \rightarrow u$ in the weak topology of $W^{1, p}(\Omega)$. When $p=\infty$, weak convergence should be replaced by weak* convergence. In what follows we shall use the abbreviation $s w l s c$ for "sequential weak lower semicontinuity", or $s w^{*} l s c$ when "weak" is replaced by "weak"”. 
On the other hand, if we allow the function $u$ to be vector-valued, i.e. $u \in$ $W^{1, p}\left(\Omega ; \mathbb{R}^{m}\right)$, then the convexity hypothesis turns out to be sufficient, but too strong to be necessary, for $F$ to be lsc: MORREY proved in [13] that, under strong regularity assumptions, $F$ is $s w^{*} l s c$ on $W^{1, \infty}\left(\Omega ; \mathbb{R}^{m}\right)$ if and only if $f$ is quasiconvex, that is for every $s \in \mathbb{R}^{m}$ and for almost every $x \in \mathbb{R}^{n}$ the function $\xi \mapsto f(x, s, \xi)$ satisfies the condition

$$
f(x, s, \xi) \cdot \operatorname{meas}(\Omega) \leqq \int_{\Omega} f(x, s, \xi+D w(y)) d y
$$

for every $\xi \in \mathbb{R}^{n m}$, for every bounded open set $\Omega \subset \mathbb{R}^{n}$, and for every $w \in$ $C_{0}^{\infty}\left(\Omega ; \mathbb{R}^{m}\right)$. Although it is technically not easy to handle, this condition arises in a natural way in many problems (especially in elastostatics); moreover, it is equivalent to convexity in $\xi$ in the case $m=1$.

The theorem of MorREY was extended by MEYERs [12] to the semicontinuity (on $W^{k, p}\left(\Omega ; \mathbb{R}^{m}\right)$ ) of functionals of the type

$$
\int_{\Omega} f\left(x, u(x), \ldots, D^{k} u(x)\right) d x,
$$

always under strong continuity hypotheses.

In section II, by means of techniques basically relying on a recent theorem of LIU [10], which allows us to deduce semicontinuity on $W^{1, p}\left(\Omega ; \mathbb{R}^{m}\right)$ from semicontinuity on $W^{1, \infty}\left(\Omega ; \mathbb{R}^{m}\right)$, we prove the following main result (theorem [II.4]) :

If $f: \mathbb{R}^{n} \times \mathbb{R}^{m} \times \mathbb{R}^{n m} \rightarrow \mathbb{R}$ satisfies

(i) $f$ is measurable in $x$, continuous in $(s, \xi)$, and

(ii) $0 \leqq f(x, s, \xi) \leqq a(x)+C\left(|s|^{p}+|\xi|^{p}\right)$,

where $p \geqq 1, a$ is a non-negative locally summable function, and $C$ is a nonnegative constant,

then the functional $(0.1)$ is swlsc on $W^{1, p}\left(\Omega ; \mathbb{R}^{m}\right)$ if and only if $\xi \mapsto f(x, s, \xi)$ is quasi-convex, for every $s$ and almost every $x$.

Counterexamples valid even in the convex case show that these hypotheses are almost the minimal ones necessary to obtain a theorem of this kind.

As a particular case of our result, we deduce the weak semicontinuity on $W^{1, n}\left(\Omega ; \mathbb{R}^{n}\right)$ of the functional

$$
\int_{\Omega} b(x)|\operatorname{det} D u(x)| d x
$$

(where $b$ is non-negative, and bounded on bounded sets of $\mathbb{R}^{n}$ ). The integrand satisfies a stronger hypothesis than quasi-convexity (namely polyconvexity, a condition introduced and studied by BALL in [2], [3], [4], [5]), but the result does not seem to be previously known.

In the last section we prove a representation theorem for the greatest swlsc functional which is less than or equal to $\int_{\Omega} f(x, u(x), D u(x)) d x$, where $f$ is not necessarily quasi-convex. We show that, under reasonable continuity assumptions on $f$, this functional has the form $\int_{\Omega} \phi(x, u(x) D u(x)) d x$, where $\phi$ is the greatest 
quasi-convex function which is less than or equal to $f$. A similar result has been proved by DACorogna [6], if $f$ is a polyconvex function.

We remark that, using more complicated notations as in [12], [5], our results can be extended to the case of functionals of the type (0.3). For other results and additional bibliography on quasi-convexity, see the many important papers by BALl, Meyers, and Morrey.

\section{Notation and Preliminary Lemmas}

If $a \in \mathbb{R}^{n}$, then $|a|$ is its euclidean norm; if $\xi$ is an $m \times n$ matrix, $|\xi|$ is the norm of $\xi$ when regarded as a vector in $\mathbb{R}^{m n}$. The Lebesgue measure of a measurable subset $S$ of $\mathbb{R}^{n}$ will be denoted by meas $(S)$.

Let $\Omega\left(\mathbb{R}^{n}\right.$ be an open set, $1 \leqq p \leqq+\infty, m \geqq 1$; we define $L^{p}\left(\Omega ; \mathbb{R}^{m}\right)$ to be the collection of all $m$-tuples $\left(f^{(1)}, \ldots, f^{(m)}\right)$ of real functions in $L^{p}(\Omega)$. Analogously, we say that $u \in W^{1, p}\left(\Omega ; \mathbb{R}^{m}\right)$ if $u$ belongs to $L^{p}\left(\Omega ; \mathbb{R}^{m}\right)$ together with its distribution derivatives $\frac{\partial u^{(i)}}{\partial x_{j}}, 1 \leqq i \leqq m, 1 \leqq j \leqq n$. The $m \times n$ matrix of these derivatives will be denoted by the symbol $D u ; W^{1, p}\left(\Omega ; \mathbb{R}^{m}\right)$ becomes a Banach space if it is endowed with the norm

$$
\|u\|_{W^{1, p\left(\Omega ; \mathrm{R}^{m}\right)}}=\|\| u\left\|_{L^{p(\Omega)}} p+\right\|\|D u\|_{L^{p}(\Omega)},
$$

where

$$
|u|(x)=|u(x)|, \quad|D u|(x)=|D u(x)| .
$$

Finally, $u \in C_{0}^{1}\left(\Omega ; \mathbb{R}^{m}\right)$ if each $u^{(i)}$ is a $C^{\mathbf{1}}$ function on $\Omega$ with compact support, while $W_{0}^{1, p}\left(\Omega ; \mathbb{R}^{m}\right)$ is the closure of $C_{0}^{1}\left(\Omega ; \mathbb{R}^{m}\right)$ in the topology of $W^{1, p}\left(\Omega ; \mathbb{R}^{m}\right)$.

Definition [I.1]. $f: \mathbb{R}^{n m} \rightarrow \mathbb{R}$ is weakly quasi-convex if for every $\tilde{\xi} \in \mathbb{R}^{n m}, \tilde{\eta} \in \mathbb{R}^{m}$ and $\tilde{\lambda} \in \mathbb{R}^{n}$ the functions

$$
\lambda \mapsto f(\tilde{\xi}+\lambda \otimes \tilde{\eta}), \quad \eta \mapsto f(\tilde{\xi}+\tilde{\lambda} \otimes \eta)
$$

are convex, where $(\lambda \otimes \eta)_{i j}=\lambda_{i} \eta_{j}$.

Definition [I.2]. A continuous function $f: \mathbb{R}^{n m} \rightarrow \mathbb{R}$ is quasi-convex if for every $\tilde{\xi} \in \mathbb{R}^{n m}$, for every open subset $\Omega$ of $\mathbb{R}^{n}$, and every function $z \in C_{0}^{1}\left(\Omega ; \mathbb{R}^{m}\right)$ we have

$$
\text { meas }(\Omega) \cdot f(\tilde{\xi}) \leqq \int_{\Omega} f(\tilde{\xi}+D z(x)) d x
$$

In what follows, if $f$ is a real function defined in $\mathbb{R}^{n} \times \mathbb{R}^{m} \times \mathbb{R}^{n m}$, we will say that $f$ is quasi convex in $\xi$ if there exists a set $I \subset \mathbb{R}^{n}$, with meas $(I)=0$, such that for every $\tilde{x} \in \mathbb{R}^{n} \backslash I$ and $\tilde{s} \in \mathbb{R}^{m}$ the function $\xi \mapsto f(\tilde{x}, \tilde{s}, \xi)$ is quasiconvex.

To prove that a function $f$ is quasi-convex, note that it is enough to verify (I.1) for one open set $\Omega$, and for every $z \in C_{0}^{\infty}\left(\Omega ; \mathbb{R}^{m}\right)$; moreover if $f$ is quasiconvex then (I.1) holds for every $z \in W_{0}^{1, \infty}\left(\Omega ; \mathbb{R}^{m}\right)$. 
Quasi-convexity implies weak quasi-convexity, which in turn implies that the function locally satisfies a Lipschitz condition. If $m=1$ or $n=1$, then quasi-convexity is equivalent to convexity; in general, a function $f \in C^{2}\left(\mathbb{R}^{n m}\right)$ is weakly quasi-convex if and only if for every $\xi \in \mathbb{R}^{m n}, \lambda \in \mathbb{R}^{n}, \eta \in \mathbb{R}^{m}$ there holds

$$
\sum_{i, j=1}^{n} \sum_{h, k=1}^{m} \frac{\partial^{2} f}{\partial \xi_{i h} \partial \xi_{j k}}(\xi) \lambda_{i} \lambda_{j} \eta_{h} \eta_{k} \geqq 0
$$

(Legendre-Hadamard condition).

The proofs of the previous remarks may be found in [2], [12], [14]. The following result ([12], Lemma 1) will be useful to disengage Definition [I.1] from the boundary condition on $z$.

Lemma [I.3]. Let $f: \mathbb{R}^{n m} \rightarrow \mathbb{R}$ be quasi-convex. For every bounded open set $\Omega \subset \mathbb{R}^{n}$ and every sequence $\left(z_{k}\right) \subset W^{1, \infty}\left(\Omega ; \mathbb{R}^{m}\right)$ which is weakly* convergent to zero, we have

$$
\operatorname{meas}(\Omega) \cdot f(\xi) \leqq \liminf _{k \rightarrow \infty} \int_{\Omega} f(\xi+D z(x)) d x
$$

for every $\xi \in \mathbb{R}^{n m}$.

Definition [I.4]. $f: \dot{\mathbb{R}}^{n} \times \mathbb{R}^{m} \times \mathbb{R}^{n m} \rightarrow \mathbb{R}$ is a Carathéodory function if the following conditions are satisfied:

for every $(s, \xi) \in \mathbb{R}^{m} \times \mathbb{R}^{n m}, x \mapsto f(x, s, \xi)$ is measurable;

for almost all $x \in \mathbb{R}^{n},(s, \xi) \mapsto f(x, s, \xi)$ is continuous.

The following result of ScorZA-DragonI ([8], page 235) characterizes the class of Carathéodory functions.

Lemma [I.5]. A mapping $f: \mathbb{R}^{n} \times \mathbb{R}^{m} \times \mathbb{R}^{n m} \rightarrow \mathbb{R}$ is a Carathéodory function if and only if for every compact set $K \subset \mathbb{R}^{n}$ and $\varepsilon>0$ there exists a compact set $K_{\varepsilon} \subset K$, with meas $\left(K \backslash K_{\varepsilon}\right)<\varepsilon$, such that the restriction off to $K_{\varepsilon} \times \mathbb{R}^{m} \times \mathbb{R}^{n m}$ is continuous.

The next lemma may be found in [7].

Lemma [I.6]. Let $G \subset \mathbb{R}^{n}$ be measurable, with meas $(G)<\infty$. Assume $\left(M_{k}\right)$ is a sequence of measurable subsets of $G$ such that, for some $\varepsilon>0$, the following estimate holds:

$$
\text { meas }\left(M_{k}\right) \geqq \varepsilon \text { for all } k \in \mathbb{N} \text {. }
$$

Then a subsequence $\left(M_{k_{h}}\right)$ can be selected such that $\bigcap_{h \in N} M_{k_{h}} \neq \emptyset$.

Lemma [I.7]. Let $\left(\phi_{k}\right)$ be a bounded sequence in $L^{1}\left(\mathbb{R}^{n}\right)$. Then to each $\varepsilon>0$ there exists a triple $\left(A_{\varepsilon}, \delta, S\right)$, where $A_{\varepsilon}$ is measurable and meas $\left(A_{\varepsilon}\right)<\varepsilon, \delta>0$, 
and $S$ is an infinite subset of $\mathbb{N}$, such that for all $k \in S$

$$
\int_{B}\left|\phi_{k}(x)\right| d x<\varepsilon
$$

whenever $B$ and $A_{\varepsilon}$ are disjoint and meas $(B)<\delta$.

Proof. We reason by contradiction. Hence we suppose that there exists a $\varepsilon>0$ such that, for every $\left(A_{\varepsilon}, \delta, S\right)$ as in the statement of the theorem, we may choose a measurable set $B$, with $B \cap A_{\varepsilon}=\emptyset$ and meas $(B)<\delta$, and an index $k \in S$ such that

$$
\int_{B}\left|\phi_{k}(x)\right| d x \geqq \varepsilon .
$$

This implies that for every set $A$, with meas $(A)<\varepsilon$, and every infinite set $S \subset \mathbb{N}$, there exists both a set $C$, with $C \cap A=\emptyset$ and meas $(A \cup C)<\varepsilon$, and an infinite subset $T$ of $S$ such that

$$
\int_{C}\left|\phi_{k}(x)\right| d x \geqq \varepsilon \quad \text { for } \quad \text { all } k \in T .
$$

This will be proved later; now we show that we are led to a contradiction.

Set $A=\emptyset, S=\mathbb{N}$, and let $C_{1}$ and $T_{1}$ be as above. Starting from $A=C_{1}$ and $S=T_{1}$, we pass to $C_{2}$ and $T_{2}$, where $C_{1} \cap C_{2}=\emptyset$ and

$$
\int_{C_{1} \cup C_{2}}\left|\phi_{k}(x)\right| d x=\int_{C_{1}}\left|\phi_{k}(x)\right| d x+\int_{C_{2}}\left|\phi_{k}(x)\right| d x \geqq 2 \varepsilon
$$

for all $k \in T_{2}$. Since meas $\left(C_{1} \cup C_{2}\right)<\varepsilon$, we may set $A=C_{1} \cup C_{2}, S=T_{2}$, and continue with the same argument. If

$$
N>\varepsilon^{-1} \sup _{k \in \mathbb{N}}\left\|\phi_{k}\right\|_{L^{1}\left(\mathrm{R}^{n}\right)},
$$

then after $N$ iterations we obtain the contradiction.

We return to the interrupted proof: let $A$ and $S$ be as stated, set $S_{1}=S$ and take $\delta_{1}<(\varepsilon-$ meas $(A)) / 2$. There exist a set $B_{1}$ disjoint from $A$, with meas $\left(B_{1}\right)<\delta_{1}$, and an index $k_{1} \in S_{1}$, such that

$$
\int_{B_{1}}\left|\phi_{k_{1}}(x)\right| d x \geqq \varepsilon .
$$

Applying induction, put

and set

$$
\delta_{n}=\frac{1}{2} \delta_{n-1}, S_{n}=\left\{k \in S_{n-1}: k>k_{n-1}\right\},
$$

$$
C=\bigcup_{h \in \mathbb{N}} B_{h}, T=\left\{k_{h}: h \in \mathbb{N}\right\},
$$

then $C$ and $T$ satisfy our requirements: $C \cap A=\emptyset$, meas $(C)+$ meas $(A)<\varepsilon$, $T \subset S$ is infinite, and

$$
\int_{C}\left|\phi_{k}(x)\right| d x \geqq \varepsilon
$$

for all $k \in T$. This completes the proof. 


$$
\begin{aligned}
& \quad \text { If } r>0 \text { and } x \in \mathbb{R}^{n}, \text { set } B_{r}(x)=\left\{y \in \mathbb{R}^{n}:|y-x|<r\right\} \text {, and meas }\left(B_{r}(x)\right) \\
& =\omega_{n} r^{n} .
\end{aligned}
$$

Definition [I.8]. Let $u \in C_{0}^{\infty}\left(\mathbb{R}^{n}\right)$. We define

$$
\left(M^{*} u\right)(x)=(M u)(x)+\sum_{i=1}^{n}\left(M D_{i} u\right)(x),
$$

where we set

$$
(M f)(x)=\sup _{r>0} \frac{1}{\omega_{n} r^{n}} \int_{B_{r}(x)}|f(y)| d y
$$

for every locally summable f.

Lemma [I.9]. If $u \in C_{0}^{\infty}\left(\mathbb{R}^{n}\right)$ then $M^{*} u \in C^{0}\left(\mathbb{R}^{n}\right)$ and

$$
|u(x)|+\sum_{i=1}^{n}\left|D_{i} u(x)\right| \leqq\left(M^{*} u\right)(x)
$$

for all $x \in \mathbb{R}^{n}$. Moreover (see [16]) if $p>1$ then

$$
\left\|M^{*} u\right\|_{L^{p}\left(\mathrm{R}^{n)}\right.} \leqq c(n, p)\|u\|_{W^{1, p_{\left(\mathrm{R}^{n}\right)}}}
$$

and if $p=1$ then

$$
\left.\operatorname{meas}\left\{x \in \mathbb{R}^{n}:\left(M^{*} u\right) \geqq \lambda\right]\right\} \leqq \frac{c(n)}{\lambda}\|u\|_{W^{1,1}\left(\mathrm{R}^{n}\right)}
$$

for all $\lambda>0$.

Lemma [I.10]. Let $u \in C_{0}^{\infty}\left(\mathbb{R}^{n}\right)$, and put

$$
U(x, y)=\frac{\left|u(y)-u(x)-\sum_{i=1}^{n} D_{i} u(x)\left(y_{i}-x_{i}\right)\right|}{|y-x|} .
$$

Then for every $x \in \mathbb{R}^{n}$ and $r>0$

$$
\int_{B_{r}(x)} U(x, y) d y \leqq 2 \omega_{n} r^{n}\left(M^{*} u\right)(x)
$$

The proof is contained in [10], Lemma 2. By modifying another demonstration of [10], we are also able to prove

Lemma [I.11]. Let $u \in C_{0}^{\infty}\left(\mathbb{R}^{n}\right)$ and $\lambda>0$, and set

$$
H^{\lambda}=\left\{x \in \mathbb{R}^{n}:\left(M^{*} u\right)(x)<\lambda\right\} .
$$

Then for every $x, y \in H^{\lambda}$ we have

$$
\frac{|u(y)-u(x)|}{|y-x|} \leqq c(n) \lambda .
$$


Proof. Let $c^{\prime}(n)$ be such that for every $x, y \in \mathbb{R}^{n}$ with $|x-y|=r$, we have

$$
\text { meas }\left(B_{r}(x) \cap B_{r}(y)\right)>\frac{2}{c^{\prime}(n)} \omega_{n} r^{n} \text {. }
$$

For $\delta>0$ set

$$
W_{r}(x, \delta)=\left\{y \in B_{r}(x): U(x, y)<\delta\right\},
$$

whence by Lemma [I.10]

$$
\operatorname{meas}\left(B_{r}(x) \backslash W_{r}(x, \delta)\right) \leqq \frac{2}{\delta} \omega_{n} r^{n}\left(M^{*} u\right)(x)
$$

If $z \in H^{\lambda}$, then

$$
\operatorname{meas}\left(B_{r}(z) \backslash W_{r}\left(z, 2 c^{\prime}(n) \lambda\right)\right) \leqq \frac{2 \omega_{n} r^{n}}{2 c^{\prime}(n) \lambda}\left(M^{*} u\right)(z)<\frac{\omega_{n} r^{n}}{c^{\prime}(n)} .
$$

Let $x, y \in H^{\lambda}$ with $r=|x-y|$. By (I.2) and (I.3)

$$
W_{r}\left(x, 2 c^{\prime}(n) \lambda\right) \cap W_{r}\left(y, 2 c^{\prime}(n) \lambda\right) \neq \emptyset .
$$

Choose $\tilde{z}$ in this intersection, so that $|\tilde{z}-x|<r,|\tilde{z}-y|<r$. Then

$$
\begin{aligned}
\frac{|u(y)-u(x)|}{|y-x|} & \leqq U(y, \tilde{z})+\sum_{i=1}^{n}\left[\left|D_{i} u(x)\right|+\left|D_{i} u(y)\right|\right] \\
& \leqq\left(4 c^{\prime}(n)+2\right) \lambda,
\end{aligned}
$$

as required.

Lemma [1.12]. Let $X$ be a metric space, $E$ a subspace of $X$, and $k$ a positive real number. Then any $k$-Lipschitz mapping from $E$ into $\mathbb{R}$ can be extended by a $k$ Lipschitz mapping from $X$ into $\mathbb{R}$.

For the proof see [8], page 298. We conclude this preliminary section by defining

$$
G^{v}=\left\{2^{-v}(x+Y): x \in \mathbb{Z}^{n}\right\}, \quad \nu \in \mathbb{N},
$$

where $Y=(0,1)^{n}=\left\{y \in \mathbb{R}^{n}: 0<y_{i}<1\right.$ for $\left.1 \leqq i \leqq n\right\}$.

\section{Semicontinuity Theorems}

If $f$ is a real function defined on $\mathbb{R}^{n} \times \mathbb{R}^{m} \times \mathbb{R}^{n m}$, and if the left hand side of (II.1) makes sense, then we define (for every measurable set $S \subset \mathbb{R}^{n}$ )

$$
\int_{S} f(x, u(x), D u(x)) d x=F(u, S) .
$$


Theorem [II.1]. Let $f: \mathbb{R}^{n} \times \mathbb{R}^{m} \times \mathbb{R}^{n m} \rightarrow \mathbb{R}$ satisfy:

(II.2) $f$ is a Carathéodory function;

(II.3) $f$ is quasi-convex in $\xi$;

(II.4) $0 \leqq f(x, s, \xi) \leqq a(x)+b(s, \xi)$ for every $x \in \mathbb{R}^{n}, \quad s \in \mathbb{R}^{m}$, and $\xi \in \mathbb{R}^{n m}$, where $a$ is a non-negative locally summable function on $\mathbb{R}^{n}$, and $b \geqq 0$ is locally bounded on $\mathbb{R}^{m} \times \mathbb{R}^{n m}$.

Then for every open set $\Omega$ in $\mathbb{R}^{n}$ the functional $u \mapsto F(u, \Omega)$ is $s w^{*} l s c$ on $W^{1, \infty}\left(\Omega ; \mathbb{R}^{m}\right)$.

Proof. Let us suppose first that $\Omega=(0,1)^{n}$. Fix $u \in W^{1, \infty}\left(\Omega ; \mathbb{R}^{m}\right)$ and $\left(z_{k}\right) \subset$ $W^{1, \infty}\left(\Omega ; \mathbb{R}^{m}\right)$ with $z^{k} \rightarrow 0$ (weak * convergence) in $W^{1, \infty}\left(\Omega ; \mathbb{R}^{m}\right)$; we must prove that

$$
F(u, \Omega) \leqq \liminf _{k \rightarrow \infty} F\left(u+z_{k}, \Omega\right) .
$$

Without loss of generality we may suppose $a(x)<+\infty$ for every $x$. Put

$$
\begin{aligned}
\lambda & =\|u\|_{W^{1, \infty}\left(\Omega ; \mathrm{R}^{m}\right)}+\sup _{k \in N}\left\|z_{k}\right\|_{W^{1, \infty}\left(\Omega ; \mathrm{R}^{m}\right)} \\
M & =\sup \{b(s, \xi):|s| \leqq \lambda,|\xi| \leqq \lambda\} .
\end{aligned}
$$

Now take $\varepsilon>0$, and let $\alpha \geqq 1$ be so large that if

$$
E=\{x \in \Omega: a(x) \leqq \alpha\} \backslash I
$$

then

$$
\operatorname{meas}(\Omega \backslash E)<\frac{\varepsilon}{M}, \quad \int_{\Omega \backslash E} a(x) d x<\varepsilon .
$$

By Lemma [I.5] there exists a compact set $K \subset \Omega$ such that $f$ is continuous on $K \times \mathbb{R}^{m} \times \mathbb{R}^{n m}$ and

$$
\operatorname{meas}(\Omega \backslash K)<\frac{\varepsilon}{\alpha+M}
$$

If we neglect sets of measure zero, then for all $\nu \in \mathbb{N}$ we can write

$$
\Omega=\bigcup_{h=1}^{2^{n v}} Q_{h}^{v}
$$

with $Q_{h}^{\nu} \in G^{\nu}$. The range $1 \leqq h \leqq 2^{n v}$ will be assumed henceforth, and we shall also write $\sum_{h}$ and $\bigcup_{h}$ when $h$ ranges from 1 to $2^{n \nu}$. Define

$$
\begin{aligned}
(u)_{h}^{v}=2^{-n v} \int_{Q_{h}^{v}} u(y) d y, & (u)^{v}(x)=\sum_{h}(u)_{h}^{v} \chi_{Q_{h}^{v}}(x) \\
(D u)_{h}^{v}=2^{-n v} \int_{\mathcal{Q}_{h}^{v}} D u(y) d y, & (D u)^{y}(x)=\sum_{h}(D u)_{h}^{v} \chi_{Q_{h}^{v}}(x) .
\end{aligned}
$$


Note that

$$
\left\|(u)^{v}\right\|_{L^{\infty}\left(\Omega ; \mathrm{R}^{m}\right)}+\left\|(D u)^{v}\right\|_{L^{\infty}\left(\Omega ; \mathrm{R}^{n m)}\right.} \leqq\|u\|_{W^{1, \infty}\left(\Omega ; \mathrm{R}^{m}\right)},
$$

and that the sequences $\left((u)^{\eta}\right)$ and $\left((D u)^{\nu}\right)$ converge pointwise a.e. to $u$ and $D u$ respectively.

For every $v$ and $h$ fix $x_{h}^{p} \in Q_{h}^{v} \cap K \cap E$, if this set is not empty. Then

$$
F\left(u+z_{k}, \Omega\right) \geqq F\left(u+z_{k}, K \cap E\right)=a_{k}+b_{k}^{v}+c_{k}^{v}+d^{v}+e,
$$

where we put

$$
\begin{aligned}
& a_{k}=\int_{K \cap E}\left[f\left(x,\left(u+z_{k}\right)(x),\left(D u+D z_{k}\right)(x)\right)-f\left(x, u(x),\left(D u+D z_{k}\right)(x)\right)\right] d x ; \\
& b_{k}^{v}=\sum_{h} \int_{Q_{h}^{v} \wedge K \cap E}\left[f\left(x, u(x),\left(D u+D z_{k}\right)(x)\right)-f\left(x_{h}^{v},(u)_{h}^{v},(D u)_{h}^{v}+D z_{k}(x)\right)\right] d x ; \\
& c_{k}^{v}=\sum_{h} \int_{Q_{h}^{v} \cap K \cap E}\left[f\left(x_{h}^{v},(u)_{h}^{v},(D u)_{h}^{v}+D z_{k}(x)\right)-f\left(x_{h}^{v},(u)_{h}^{v},(D u)_{h}^{v}\right)\right] d x ; \\
& d^{v}=\sum_{h} \int_{Q_{h}^{v} \wedge K \cap E}\left[f\left(x_{h}^{v},(u)_{h}^{v},(D u)_{h}^{v}\right)-f(x, u(x), D u(x))\right] d x .
\end{aligned}
$$

By the uniform continuity of $f$ on the bounded sets of $K \times \mathbb{R}^{n} \times \mathbb{R}^{n m}$ we have $\lim _{k \rightarrow \infty} a_{k}=0$. Similarly the uniform continuity of $f$ and the pointwise convergence of $\left((u)^{v}\right)$ and $\left((D u)^{n}\right)$ imply that

$$
\lim _{\nu \rightarrow \infty} d^{v}=0, \quad \lim _{\nu \rightarrow \infty} b_{k}^{v}=0 \text { uniformly with respect to } k .
$$

Hence we may suppose that $v$ is large enough to ensure that $\left|b_{k}^{v}\right|+\left|d^{v}\right|<\varepsilon$ for all $k$.

Now note that

$$
\begin{aligned}
\mid \sum_{h} \int_{Q_{h}^{v} \backslash(K \cap E)} & {\left[f\left(x_{h}^{v},(u)_{h}^{v},(D u)_{h}^{v}+D z_{k}(x)\right)-f\left(x_{h}^{v},(u)_{h}^{v},(D u)_{h}^{v}\right)\right] d x \mid } \\
& \leqq 2 \sum_{h} \int_{Q_{h}^{v} \backslash(K \cap E)}\left[a\left(x_{h}^{v}\right)+M\right] d x \\
& \leqq 2\left[(\alpha+M) \text { meas }(\Omega \backslash K)+M \text { meas }(\Omega \backslash E)+\int_{\Omega \backslash E} \alpha d x\right] \\
& \leqq 4 \varepsilon+2 \int_{\Omega \backslash E} a(x) d x \leqq 6 \varepsilon .
\end{aligned}
$$

Applying Lemma [I.3] to each $Q_{h}^{v}$, we find that

$$
\liminf _{k \rightarrow \infty} c_{k}^{\prime \prime} \geqq-6 \varepsilon \text {. }
$$

Finally,

$$
e=F(u, K \cap E) \geqq F(u, \Omega)-3 \varepsilon .
$$

As $k \rightarrow \infty$, the foregoing estimates yield

$$
\liminf _{k \rightarrow \infty} F\left(u+z_{k}, \Omega\right) \geqq F(u, \Omega)-10 \varepsilon,
$$


Since $\varepsilon$ was arbitrary, this proves our result for the special choice of $\Omega$ noted at the beginning.

It is easy to see that the same argument applies to every hypercube $\Omega$ with edges parallel to the coordinate axes; the assertion for a generic $\Omega$ follows from the fact that the supremum of a family of 1sc functions is 1sc. This completes the proof.

A slight modification of the proof yields the same theorem even if $f$ satisfies (II.2) and (II.3), and $|f|$ satisfies (II.4) (see [12], [9]). Note that if $f$ is defined on $\Omega \times \mathbb{R}^{m} \times\left\{\xi \in \mathbb{R}^{n m}:|\xi|<r\right\}$ for some $r>0$ and the hypotheses of theorem [II.1] hold, then the functional $u \mapsto F(u, \Omega)$ is sw*Isc on the space of functions $u \in$ $W^{1, \infty}\left(\Omega ; \mathbb{R}^{m}\right)$ such that $\|D u\|_{L^{\infty}\left(\Omega ; \mathbb{R}^{n m}\right)}<r$.

The inverse to theorem [II.1] is given by

Theorem [II.2]. Let $f: \mathbb{R}^{n} \times \mathbb{R}^{m} \times \mathbb{R}^{n m} \rightarrow \mathbb{R}$ satisfy (II.2) and (II.4). Assume the functional $u \mapsto F(u, \Omega)$ to be sw*lsc on $W^{1, \infty}\left(\Omega ; \mathbb{R}^{m}\right)$ for every open set $\Omega \subset \mathbb{R}^{n}$. Then $f$ is quasi-convex in $\xi$.

Proof. We have to show that, if we fix an open set $\Omega\left(\mathbb{R}^{n}\right.$, then there exists a set $I \subset \Omega$, with meas $(I)=0$, such that $\xi \mapsto f(x, s, \xi)$ is quasi-convex for every $x \in \Omega \backslash I$ and $s \in \mathbb{R}^{m}$. To this end, we will use only the fact that $u \mapsto F(u, \Omega)$ is lsc for that particular $\Omega$.

By Lemma [I.5] we can choose a nondecreasing sequence $\left(K_{i}\right)$ of compact sets, with meas $\left(\Omega \backslash K_{i}\right)<\frac{1}{i}$, such that $f$ is continuous on each $K_{i} \times \mathbb{R}^{m} \times \mathbb{R}^{n m}$.

Define $I$ in the following way: $x \in \Omega \backslash I$ if the following conditions are satisfied:

$$
\begin{gathered}
\quad x \in \bigcup_{i \in \mathbb{N}} K_{i} ; \\
a(x)<+\infty ; \\
x \text { is a Lebesgue point for } \chi_{K_{i}} \text {, for every } i \text {;* } \\
x \text { is a Lebesgue point for } a \cdot \chi_{\Omega \backslash K_{i}} \text { for every } i .
\end{gathered}
$$

Fix $\tilde{x} \in \Omega \backslash I, \tilde{s} \in \mathbb{R}^{m}, \tilde{\xi} \in \mathbb{R}^{n m}$, where clearly we may suppose $\tilde{x}=0$, and also set $u(x)=\tilde{s}+\tilde{\xi} \cdot x$, where $\tilde{\xi}$ is regarded as an $m \times n$ matrix. Let $z \in$ $C_{0}^{\infty}\left(Y ; \mathbb{R}^{m}\right)$, and put

$$
\lambda=\|u\|_{W^{1, \infty}\left(\Omega ; \mathrm{R}^{m}\right)}+\|z\|_{W^{1, \infty}\left(y ; \mathrm{R}^{m}\right)} .
$$

Define $z$ periodically on $\mathbb{R}^{n}$, setting $z(x)=z(x+y)$ for every $y \in \mathbb{Z}^{n}$. Let $\tilde{k}$ be so large that $2^{-\widetilde{k}} Y \subset \Omega$; for $k \geqq \widetilde{k}$ and $\nu \in \mathbb{N}$ define

$$
z_{k}^{v}(x)=\left\{\begin{array}{l}
2^{-k v} z\left(2^{k v} x\right) \quad \text { if } \quad x \in 2^{-k} Y \\
0 \quad \text { otherwise, }
\end{array}\right.
$$

* This means that $\lim _{r \rightarrow 0}\left[\text { meas }\left(B_{r}(x)\right)\right]^{-1} \int_{B_{r}(x)} \chi_{K_{i}}(y) d y=1$. 
so that $\left\|z_{k}^{v}\right\|_{W^{1, \infty}\left(\Omega ; \mathrm{R}^{m}\right)} \leqq \lambda$. For every $k, z_{k}^{v} \rightarrow 0$ (weak*) in $W^{1, \infty}\left(\Omega ; \mathbb{R}^{m}\right)$ as $\nu \rightarrow+\infty$, hence $z_{k}^{v} \rightarrow 0$ strongly in $L^{\infty}\left(\Omega ; \mathbb{R}^{m}\right)$. Also for fixed $k$ if we neglect sets of measure 0 , then

$$
2^{-k} Y=\bigcup_{h} Q_{h}^{k v}
$$

with $Q_{h}^{k \nu} \in G^{k \nu}$ for $1 \leqq h \leqq 2^{n \nu}$. We denote by $x_{h}^{\nu}$ the corner of $Q_{h}^{k v}$ nearest to the origin, so that $Q_{h}^{k v}=x_{h}^{y}+2^{-k v} Y$.

By (II.5), we may suppose that $0 \in K_{i}$ for all $i$. Choose $\varepsilon>0$. Then there exists $\tilde{i}$ such that for $i \geqq \tilde{i}$ we have

$$
\int_{\Omega \backslash K_{i}}[a(x)+M] d x<\varepsilon
$$

where

$$
M=\sup \{b(s, \xi):|s|+|\xi| \leqq 2 \lambda\} .
$$

Let $\tilde{f}_{i}$ be a continuous function on $\mathbb{R}^{n} \times \mathbb{R}^{m} \times \mathbb{R}^{n m}$, coinciding with $f$ on $K_{i} \times\{(s, \xi):|s|+|\xi| \leqq 2 \lambda\}=K_{i} \times B_{2 \lambda}$. We may also suppose that $\tilde{f}_{i}$ satisfies $0 \leqq \tilde{f}_{i} \leqq \max _{K_{i} \times B_{2 \lambda}} f$. Choose a function $\psi \in C_{0}^{0}(\Omega)$ so that

$$
\begin{gathered}
0 \leqq \psi(x) \leqq 1 \text { for all } x \in \Omega, \\
\psi(x)=1 \text { for all } x \in K_{i}, \\
\int_{\Omega \backslash K_{i}} \psi(x) d x<\varepsilon / \max _{K_{i} \times B_{2 \lambda}} f .
\end{gathered}
$$

The function $f_{i}=\psi \tilde{f_{i}}$ is another continuous extension of $f$ outside $K_{i} \times B_{2 \lambda}$. We can split the functional $F\left(u+z_{k}^{\nu}, 2^{-k} Y\right)$ as follows:

$$
F\left(u+z_{k}^{y}, 2^{-k} Y\right)=a^{p}+b^{\nu}+c^{\nu},
$$

where we set

$$
\begin{aligned}
& a^{v}=\int_{2^{-k_{Y}}}\left[f\left(x,\left(u+z_{k}^{\nu}\right)(x),\left(D u+D z_{k}^{\nu}\right)(x)\right)\right. \\
& \left.-f_{i}\left(x,\left(u+z_{k}^{\nu}\right)(x),\left(D u+D z_{k}^{\nu}\right)(x)\right)\right] d x ; \\
& b^{\nu}=\sum_{h} \int_{Q_{h}^{k v}}\left[f_{i}\left(x,\left(u+z_{k}^{v}\right)(x),\left(D u+D z_{k}^{v}\right)(x)\right)\right. \\
& \left.-f_{i}\left(x_{h}^{v}, u\left(x_{h}^{v}\right), D u\left(x_{h}^{v}\right)+D z_{k}^{v}(x)\right)\right] d x ; \\
& c^{v}=\sum_{h} \int_{Q_{h}^{k \nu}} f_{i}\left(x_{h}^{\nu}, u\left(x_{h}^{\nu}\right), D u\left(x_{h}^{\nu}\right)+D z\left(2^{k v} x\right)\right) d x \\
& =\sum_{h} 2^{-n k v} \int_{Y} f_{i}\left(x_{h}^{v}, u\left(x_{h}^{\nu}\right), D u\left(x_{h}^{\nu}\right)+D z(y)\right) d y .
\end{aligned}
$$

Our choice of $f_{i}$ yields $\left|a^{v}\right|<2 \varepsilon$ for every $v$ and $i \geqq \tilde{i}$. Moreover since $u \in$ $C^{1}\left(\bar{\Omega} ; \mathbb{R}^{m}\right)$ and $f_{i}$ is uniformly continuous, we have $\lim _{\nu \rightarrow \infty} b^{v}=0$. Finally $c^{v}$ has 
the form of a Cauchy sum, over the cube $2^{-k} Y$, of the continuous function

$$
x \mapsto \int_{Y} f_{i}(x, u(x), D u(x)+D z(y)) d y .
$$

Hence it is convergent as $v \rightarrow \infty$, with

$$
\lim _{\nu \rightarrow \infty} c^{v}=\int_{2^{-k_{Y}}}\left[\int_{Y} f_{i}(x, u(x), D u(x)+D z(y)) d y\right] d x .
$$

Combining the above three lines we have

$$
\limsup _{\nu \rightarrow \infty} F\left(u+z_{k}^{\nu}, 2^{-k} Y\right) \leqq 2 \varepsilon+\int_{2^{-k_{Y}}}\left[\int_{Y} f_{i}(x, u(x), D u(x)+D z(y)) d y\right] d x .
$$

Let $\psi$ tend to $\chi_{K_{i}}$. Since $f=f_{i}$ on $K_{i} \times B_{2 \lambda}$, it follows from the dominated convergence theorem that

$$
\limsup _{y \rightarrow \infty} F\left(u+z_{k}^{p}, 2^{-k} Y\right) \leqq 2 \varepsilon+\int_{K_{i} \cap 2^{-k_{Y}}}\left[\int_{Y} f(x, u(x), D u(x)+D z(y)) d y\right] d x .
$$

By the semicontinuity of $u \mapsto F(u, \Omega)$ and the fact that $z_{k}^{p} \equiv 0$ on $\Omega \backslash 2^{-k} Y$

$$
\begin{aligned}
F(u, \Omega)= & F\left(u, 2^{-k} Y\right)+F\left(u, \Omega \backslash 2^{-k} Y\right) \\
& \leqq \liminf _{v \rightarrow \infty}\left[F\left(u+z_{k}^{v}, 2^{-k} Y\right)+F\left(u, \Omega \backslash 2^{-k} Y\right)\right] .
\end{aligned}
$$

Hence for $i \geqq \tilde{i}$

$$
F\left(u, 2^{-k} Y\right) \leqq 2 \varepsilon+\int_{2^{-k_{Y \times Y}}} \chi_{K_{i}}(x) f(x, u(x), D u(x)+D z(y)) d x d y .
$$

Letting $i \rightarrow+\infty$, and using the fact that $\varepsilon$ is arbitrary, we get

$$
F\left(u, 2^{-k} Y\right) \leqq \int_{2^{-k_{Y}}}\left[\int_{Y} f(x, u(x), D u(x)+D z(y)) d y\right] d x,
$$

so that

$$
2^{n k} \int_{2^{-k_{Y}}}\left[f(x, u(x), D u(x))-\int_{Y} f(x, x, u(x), D u(x)+D z(y)) d y\right] d x \leqq 0 .
$$

Call $\mu(x ; u, z)$ the integrand in the square brackets; our hypotheses on the set $I$, and the continuity of $f$ on $K_{\tilde{i}} \times B_{2 \lambda}$, then yield

$$
\begin{aligned}
\lim _{k \rightarrow \infty} 2^{n k} & \int_{2^{-k} Y \cap K_{\tilde{i}}} \mu(x ; u, z) d x \\
& =\lim _{k \rightarrow \infty}\left(2^{n k} \int_{2^{-k_{Y}}} \chi_{K_{\tilde{i}}}(x) d x\right)\left(\left[\operatorname{meas}\left(2^{-k} Y \cap K_{\tilde{i}}\right)\right]^{-1} \int_{2^{-k} \int_{Y \cap K_{\tilde{i}}}} \mu(x ; u, z) d x\right) \\
& =f(0, \tilde{s}, \tilde{\xi})-\int_{\tilde{Y}} f(0, \tilde{s}, \tilde{\xi}+D z(y)) d y .
\end{aligned}
$$


On the other hand the integral of $\mu$ on $2^{-k} Y \backslash K_{\tilde{i}}$ is small because

$$
\begin{aligned}
\left|2^{n k} \int_{2^{-k} Y \backslash K_{\tilde{i}}} \mu(x ; u, z) d x\right| & \leqq 2^{n k} \int_{2^{-k_{Y \backslash K_{\tilde{i}}}}}[a(x)+M] d x \\
& =2^{n k} \int_{2^{-k_{Y}}}[a(x)+M] \chi_{\Omega \backslash K_{\tilde{i}}}(x) d x,
\end{aligned}
$$

which tends to zero as $k \rightarrow \infty$. These estimates show that (I.1) is satisfied on the open set $Y$, hence on every open set $\Omega \subset \mathbb{R}^{n}$.

Note that the proof remains almost unchanged if we suppose that (II.2) holds, that $|f|$ satisfies (II.4), and that the functional $u \mapsto F(u, \Omega)$ is sw*Isc on each Dirichlet class $\tilde{u}+W_{0}^{1, \infty}\left(\Omega ; \mathbb{R}^{m}\right)$, with $\tilde{u}$ a polynomial of degree one.

Remark [II.3]. Let $f$ satisfy (II.2) and(II.4). Assume that the functional $u \mapsto F(u, \Omega)$ is $s w^{*} l s c$ on the space of functions $u$ in $W^{1, \infty}\left(\Omega ; \mathbb{R}^{m}\right)$ such that $\|D u\|_{L^{\infty}\left(\Omega ; \mathbb{R}^{n m}\right)}<r$ (where $r>0$ ). Then there exists a set $I \subset \Omega$, with meas $(I)=0$, such that for every $\tilde{x} \in \Omega \backslash I, \tilde{s} \in \mathbb{R}^{m}$, and $\tilde{\xi} \in B_{r}(0) \subset \mathbb{R}^{n m}$, and for every $z \in W_{0}^{1, \infty}\left(\Omega ; \mathbb{R}^{m}\right)$ such that $\|\tilde{\xi}+D z\|_{L^{\infty}\left(\Omega ; \mathbb{R}^{n m}\right)}<r$, we have

$$
\operatorname{meas}(\Omega) \cdot f(\tilde{x}, \tilde{s}, \tilde{\xi}) \leqq \int_{\Omega} f(\tilde{x}, \tilde{s}, \tilde{\xi}+D z(x)) d x
$$

Theorems [II.1] and [II.2] generalize results contained in [12], [9]. Our next theorem deals with semicontinuity in $W^{1, p}, p \geqq 1$.

Theo rem [II.4]. Let $1 \leqq p<+\infty$, and assume that $f: \mathbb{R}^{n} \times \mathbb{R}^{m} \times \mathbb{R}^{n m} \rightarrow \mathbb{R}$ satisfies (II.2), (II.3) and

(II.6) $0 \leqq f(x, s, \xi) \leqq a(x)+C\left(|s|^{p}+|\xi|^{p}\right)$ for every $x \in \mathbb{R}^{n}, \quad s \in \mathbb{R}^{m}, \xi \in \mathbb{R}^{n m}$, where $C$ is a non-negative constant and $a$ is a non-negative locally summable function on $\mathbb{R}^{n}$.

Then for every open set $\Omega \subset \mathbb{R}^{n}$ the functional $u \mapsto F(u, \Omega)$ is swlsc on $W^{1, p}\left(\Omega ; \mathbb{R}^{m}\right)$.

Proof. As in theorem [II.1] we may confine ourselves to a particular set $\Omega$, say a ball. Take $u \in W^{1, p}\left(\Omega ; \mathbb{R}^{m}\right)$ and $\left(z_{k}\right) \subset W^{1, p}\left(\Omega ; \mathbb{R}^{m}\right)$ with $z_{k} \rightarrow 0$ (weakly) in $W^{1, p}\left(\Omega ; \mathbb{R}^{m}\right)$. We may suppose

$$
\liminf _{k \rightarrow \infty} F\left(u+z_{k}, \Omega\right)=\lim _{k \rightarrow \infty} F\left(u+z_{k}, \Omega\right) \text {. }
$$

This will allow us to select subsequences without altering $\liminf _{k \rightarrow \infty} F\left(u+z_{k}, \Omega\right)$; hence we need not indicate subsequences, denoting all of them with the same index $k$.

By an extension theorem ([1], Theorem 4.26) we may assume each $z_{k}$ to be

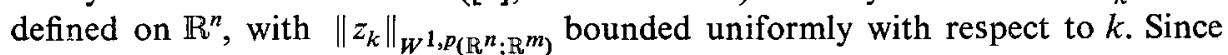
$C_{0}^{\infty}\left(\mathbb{R}^{n} ; \mathbb{R}^{m}\right)$ is dense in $W^{1, p}\left(\mathbb{R}^{n} ; \mathbb{R}^{m}\right)$ and $u \mapsto F(u, \Omega)$ is continuous in the strong topology of $W^{1, p}\left(\Omega ; \mathbb{R}^{n t}\right)$, there exists a sequence $\left(w_{k}\right) C C_{0}^{\infty}\left(\mathbb{R}^{n} ; \mathbb{R}^{m}\right)$ such that

$$
\left\|w_{k}-z_{k}\right\|_{W^{1, p}\left(\mathrm{R}^{n} ; \mathrm{R}^{m}\right)}<\frac{1}{k}, \quad\left|F\left(u+w_{k}, \Omega\right)-F\left(u+z_{k}, \Omega\right)\right|<\frac{1}{k} .
$$


Hence we may assume the sequence $\left(z_{k}\right)$ to be in $C_{0}^{\infty}\left(\mathbb{R}^{n} ; \mathbb{R}^{m}\right)$, and to be bounded in $W^{1, p}\left(\mathbb{R}^{n} ; \mathbb{R}^{m}\right)$.

Let $\eta: \mathbb{R}^{+} \rightarrow \mathbb{R}^{+}$be a continuous increasing function, with $\eta(0)=0$, such that for every measurable set $B \subset \Omega$

$$
\int_{B}\left[a(x)+C\left(|u(x)|^{p}+|D u(x)|^{p}\right)\right] d x<\eta(\text { meas }(B)) .
$$

Fix $\varepsilon>0$, and apply Lemma [I.7] to each of the $m$ sequences $\left(\left(M^{*} z_{k}^{(i)}\right)^{p}\right)$, $1 \leqq i \leqq m$. This gives a subsequence $\left(z_{k}\right)$, a set $A_{\varepsilon} \subset \Omega$, with meas $\left(A_{\varepsilon}\right)<\varepsilon$, and a real number $\delta>0$ such that

$$
\int_{B}\left[\left(M^{*} z_{k}^{(i)}\right)(x)\right]^{p} d x<\varepsilon
$$

for all $k$, for $1 \leqq i \leqq m$, and for every $B \subset \Omega \backslash A_{\varepsilon}$ with meas $(B)<\delta$. By Lemma [I.9] we may take $\lambda>0$ so large that for all $i, k$

$$
\text { meas }\left\{x \in \mathbb{R}^{n}:\left(M^{*} z_{k}^{(i)}\right)(x) \geqq \lambda\right\}<\min (\varepsilon, \delta) .
$$

For all $i, k$ set

$$
H_{i, k}^{\lambda}=\left\{x \in \mathbb{R}^{n}:\left(M^{*} z_{k}^{(i)}\right)(x)<\lambda\right\}, \quad H_{k}^{\lambda}=\bigcap_{i=1}^{m} H_{i, k}^{\lambda} .
$$

Lemma [I.11] ensures that, for all $x, y \in H_{k}^{\lambda}$ and $1 \leqq i \leqq m$,

$$
\frac{\left|z_{k}^{(i)}(y)-z_{k}^{(i)}(x)\right|}{|y-x|} \leqq c(n) \lambda .
$$

Let $g_{k}^{(i)}$ be a Lipschitz function extending $z_{k}^{(i)}$ outside $H_{k}^{\lambda}$, with Lipschitz constant not greater than $c(n) \lambda$ (Lemma [I.12]). Since $H_{k}^{\lambda}$ is an open set we have

$$
g_{k}^{(i)}(x)=z_{k}^{(i)}(x), \quad D g_{k}^{(i)}(x)=D z_{k}^{(i)}(x)
$$

for all $x \in H_{k}^{\lambda}$, and

$$
\left\|D g_{k}^{(i)}\right\|_{L^{\infty}\left(\mathrm{R}^{n)}\right.} \leqq c(n) \lambda
$$

We may also assume

$$
\left\|g_{k}^{(i)}\right\|_{L^{\infty}\left(\mathrm{R}^{n}\right)} \leqq\left\|z_{k}^{(i)}\right\|_{L^{\infty}\left(H_{k}^{\lambda}\right)} \leqq \lambda
$$

We may suppose that, at least for a subsequence,

$$
g_{k}^{(i)}-v^{(i)}\left(\text { weak }^{*}\right) \text { in } \mathrm{W}^{1, \infty}(\Omega)
$$

for $1 \leqq i \leqq m$. Put $\left(g_{k}^{(1)}, \ldots, g_{k}^{(m)}\right)=g_{k}, \quad\left(v^{(1)}, \ldots, v^{(m)}\right)=v$; we have

$$
\begin{aligned}
F\left(u+z_{k}, \Omega\right) & \geqq F\left(u+g_{k},\left(\Omega \backslash A_{\varepsilon}\right) \cap H_{k}^{\lambda}\right) \\
& =F\left(u+g_{k}, \Omega \backslash A_{\varepsilon}\right)-F\left(u+g_{k},\left(\Omega \backslash A_{\varepsilon}\right) \backslash H_{k}^{\lambda}\right) .
\end{aligned}
$$

Since

$$
\operatorname{meas}\left[\left(\Omega \backslash A_{\varepsilon}\right) \backslash H_{k}^{\lambda}\right] \leqq \sum_{i=1}^{m} \operatorname{meas}\left[\left(\Omega \backslash A_{\varepsilon}\right) \backslash H_{i, k}^{\lambda}\right]<m \min (\varepsilon, \delta)
$$


by (II.6) and by our choice of $A_{\varepsilon}$ we obtain

$$
\begin{aligned}
F\left(u+g_{k},\left(\Omega \backslash A_{\varepsilon}\right) \backslash H_{k}^{\lambda}\right) & \leqq 2^{p-1}\left\{\eta(m \varepsilon)+c(n, \Omega) \lambda^{p} \text { meas }\left[\left(\Omega \backslash A_{\varepsilon}\right) \backslash H_{k}^{\lambda}\right]\right\} \\
& \leqq 2^{p-1}\left\{\eta(m \varepsilon)+c(n, \Omega) \sum_{i=1}^{m} \int_{\left(\Omega \backslash A_{\varepsilon} \backslash H_{i, k}^{\lambda}\right.}\left[\left(M^{*} z_{k}^{(i)}\right)(x)\right]^{p} d x\right\} \\
& \leqq 2^{p-1}\{\eta(m \varepsilon)+m c(n, \Omega) \varepsilon\}=O(\varepsilon)
\end{aligned}
$$

Thus

$$
F\left(u+z_{k}, \Omega\right) \geqq F\left(u+g_{k}, \Omega \backslash A_{\varepsilon}\right)-O(\varepsilon) .
$$

Choose an open set $\Omega^{\prime} \subset \Omega$ containing $\Omega \backslash A_{\varepsilon}$ and such that

$$
\left|F\left(u+g_{k}, \Omega^{\prime}\right)-F\left(u+g_{k}, \Omega \backslash A_{\varepsilon}\right)\right|<\varepsilon
$$

(this is possible since the functions $g_{k}$ are uniformly bounded in $W^{1, \infty}\left(\Omega ; \mathbb{R}^{m}\right)$ ).

Applying Theorem [II.1] to the functional

$$
\Gamma(w, S)=\int_{S} \gamma(x, w(x), D w(x) d x),
$$

where

$$
\gamma(x, s, \xi)=f(x, u(x)+s, D u(x)+\xi),
$$

we are led to

$$
\begin{aligned}
\lim _{k \rightarrow \infty} F\left(u+z_{k}, \Omega\right) & \geqq \liminf _{k \rightarrow \infty} F\left(u+g_{k}, \Omega^{\prime}\right)-\varepsilon-O(\varepsilon) \\
& \geqq F\left(u+v, \Omega^{\prime}\right)-\varepsilon-O(\varepsilon) .
\end{aligned}
$$

At least for a subsequence we may suppose that $z_{k}(x) \rightarrow 0$ for almost all $x \in \Omega$. Set

$$
G=\{x \in \Omega: v(x) \neq 0\}
$$

and

$$
\tilde{G}=G \cap\left\{x \in \Omega: z_{k}(x) \rightarrow 0\right\},
$$

so that meas $(G)=\operatorname{meas}(\tilde{G})$. Since the functions $g_{k}$ are continuous and converge to $v$ in $L^{\infty}$, we have

$$
g_{k}(x) \rightarrow v(x)
$$

for all $x \in \Omega$, hence for all $x \in G$. If we now suppose

$$
\text { meas }(G)>(m+1) \varepsilon
$$

we obtain a contradiction. Indeed by (II.7)

$$
\text { meas }\left(\tilde{G} \cap H_{k}^{\lambda}\right)>\varepsilon \text { for all } k,
$$

and by Lemma [I.6], for a subsequence,

$$
\left(\bigcap_{h \in \mathbb{N}} H_{k_{h}}^{\lambda}\right) \cap \tilde{G} \neq \emptyset .
$$


If $\tilde{x}$ belongs to this set, then

$$
v(\tilde{x})=\lim _{h \rightarrow \infty} g_{k_{h}}(\tilde{x})=\lim _{h \rightarrow \infty} z_{k_{h}}(\tilde{x})=0,
$$

contrary to the definition of $G$.

We may thus write, by the positivity of $f$,

$$
\begin{aligned}
\lim _{k \rightarrow \infty} F\left(u+z_{k}, \Omega\right) & \geqq F\left(u, \Omega^{\prime} \backslash G\right)-O(\varepsilon)-\varepsilon \\
& \geqq F(u, \Omega)-O(\varepsilon)-\varepsilon-\eta[(m+2) \varepsilon],
\end{aligned}
$$

which concludes the proof since $\varepsilon$ is arbitrary.

In this proof the role played by the hypothesis $f \geqq 0$ is fundamental. Indeed if (II.6) is changed to

$$
|f(x, s, \xi)| \leqq a(x)+C\left(|s|^{p}+|\xi|^{p}\right),
$$

and (II.2), (II.3) are satisfied, then Theorem [II.4] is false, at least for $n>2$, but one can prove that for all $\varepsilon>0$ the funtional $u \mapsto F(u, \Omega)$ is swlsc on $W^{1, p+\varepsilon}\left(\Omega ; \mathbb{R}^{m}\right)$ (see [9]).

Since semicontinuity on $W^{1, p}$ implies semicontinuity on $W^{1, \infty}$, we may summarize the results of this section as follows:

Statement [I.5]. Let $f: \mathbb{R}^{n} \times \mathbb{R}^{m} \times \mathbb{R}^{n m} \rightarrow \mathbb{R}$ be a Carathéodory function which satisfies (II.6) for some $p \geqq 1 \quad$ [or alternately satisfies (II.4)]. Then the functional $u \mapsto F(u, \Omega)$ is swlsc on $W^{1, p}\left(\Omega ; \mathbb{R}^{m}\right)$ [or is $s w^{*} l s c$ on $\left.W^{1, \infty}\left(\Omega ; \mathbb{R}^{m}\right)\right]$ if and only if $f$ is quasi-convex in $\xi$.

\section{A Representation Theorem}

In this section, given a functional of the type (II.1) with $f$ not necessarily quasi-convex in $\xi$, we deal with the problem of finding its lsc envelope on $W^{1, p}(\Omega ; \mathbb{R})$, i.e. the greatest functional less than or equal to $F$ which is $\operatorname{swlsc}$ on $W^{1, p}\left(\Omega ; \mathbb{R}^{m}\right)$. As a consequence of statement [II.5], it will suffice to treat the case $p=+\infty$.

Let $f: \mathbb{R}^{n} \times \mathbb{R}^{m} \times \mathbb{R}^{n m} \rightarrow \mathbb{R}$ be a Carathéodory function satisfying (II.4). For every $r>0$ and for every $\Omega$ bounded open set of $\mathbb{R}^{n}$, if $u \in W^{1, \infty}\left(\Omega ; \mathbb{R}^{m}\right)$ with $\|D u\|_{L^{\infty}\left(\Omega ; R^{n m}\right)} \leqq r$, we define

$$
\begin{gathered}
F(r, u, \Omega)=\inf \left\{\liminf _{k \rightarrow \infty} F\left(u_{k}, \Omega\right): u_{k} \rightarrow u\left(\text { weak }^{*}\right) \text { in } W^{1, \infty}\left(\Omega ; \mathbb{R}^{m}\right)\right. \\
\text { and } \left.\left\|D u_{k}\right\|_{L^{\infty}\left(\Omega ; \mathrm{R}^{n m}\right)} \leqq r\right\} \\
F_{0}(r, u, \Omega)=\inf \left\{\liminf _{k \rightarrow \infty} F\left(u_{k}, \Omega\right):\left(u_{k}-u\right) \rightarrow 0\left(\text { weak }^{*}\right) \text { in } W_{0}^{1, \infty}\left(\Omega ; \mathbb{R}^{m}\right)\right. \\
\text { and } \left.\left\|D u_{k}\right\|_{L^{\infty}\left(\Omega ; \mathrm{R}^{n m}\right)} \leqq r\right\},
\end{gathered}
$$

where $F(u, \Omega)$ is defined by (II.1). The argument employed in [11], Lemmas 3.3 and 4.5 , leads us to the following results. 
Lemma [III.1]. If $f$ satisfies the foregoing hypotheses, then for every $r>0$ and every $u \in W^{1, \infty}\left(\Omega ; \mathbb{R}^{m}\right)$ with $\|D u\|_{L^{\infty}\left(\Omega ; R^{n m}\right)}<r$ there exists afunction $h_{u} \in L^{1}(\Omega)$ such that

$$
F\left(r, u, \Omega^{\prime}\right)=F_{0}\left(r, u, \Omega^{\prime}\right)=\int_{\Omega^{\prime}} h_{u}(x) d x
$$

for every open set $\Omega^{\prime} \subset \Omega$.

Lemma [II.2]. Let $u_{1}, u_{2} \in W^{1, \infty}\left(\Omega ; \mathbb{R}^{m}\right)$, with $\left\|D u_{i}\right\|_{L^{\infty}\left(\Omega ; \mathrm{R}^{n m}\right)}<r \quad$ and $\left\|u_{i}\right\|_{L^{\infty}\left(\Omega ; \mathbb{R}^{m}\right)}<d, i=1,2$. Then for every open set $\Omega^{\prime} C \Omega$ we have

$$
\left|F\left(r, u_{1}, \Omega^{\prime}\right)-F\left(r, u_{2}, \Omega^{\prime}\right)\right| \leqq \int_{\Omega^{\prime}} \omega\left(x, d, 3 r,\left\|u_{1}-u_{2}\right\|_{W^{1, \infty}\left(\Omega ; \mathbb{R}^{m}\right)}\right) d x,
$$

where

$$
\begin{gathered}
\omega(x, d, r, \delta)=\sup \left\{\left|f\left(x, s_{1}, \xi_{1}\right)-f\left(x, s_{2}, \xi_{2}\right)\right|:\left|s_{i}\right|<d,\left|\xi_{i}\right|<r \text { for } i=1,2,\right. \\
\text { and } \left.\left|s_{1}-s_{2}\right|+\left|\xi_{1}-\xi_{2}\right|<\delta\right\} .
\end{gathered}
$$

We now use these results to prove

Lemma [III.3]. To each $r>0$ there exists a Carathéodory function $\phi_{r}$ defined on $\Omega \times \mathbb{R}^{m} \times\left\{\xi \in \mathbb{R}^{n m}:|\xi|<r\right\}$ such that for every $u \in W^{1, \infty}\left(\Omega ; \mathbb{R}^{m}\right)$ with $\|D u\|_{L^{\infty}\left(\Omega ; \mathrm{R}^{n m}\right)}<r$ we have

$$
\phi_{r}(x, u(x), D u(x))=h_{u}(x) \text { for almost every } x \in \Omega \text {. }
$$

Proof. Let $\mathscr{A}_{r}$ be the class of all affine functions on $\mathbb{R}^{n}$ with rational coefficients and with gradient less than $r$ in norm. Also let $L$ be the set of the points in $\mathbb{R}^{n}$ which are Lebesgue points for every function $h_{u}$, with $u \in \mathscr{A}_{r}$. For $x \in L, s \in \mathbb{Q}^{m}$, $\xi \in \mathbb{Q}^{n m}$, with $|\xi|<r$, put

$$
\phi_{r}(x, s, \xi)=h_{u}(x),
$$

where $u \in \mathscr{A}_{r}, u(x)=s, D u=\xi$. Lemma [III.2] implies that $\phi_{r}$ is continuous in $(s, \xi)$ for almost every $x \in L$. Since $L \times \mathbb{Q}^{m} \times \mathbb{Q}^{n m}$ is dense in $L \times \mathbb{R}^{m} \times \mathbb{R}^{n m}$, we may therefore extend the definition of $\phi_{r}$ to $\Omega \times \mathbb{R}^{m} \times\left\{\xi \in \mathbb{R}^{n m}:|\xi|<r\right\}$, obtaining

$$
\left|\phi_{r}\left(x, s_{1}, \xi_{1}\right)-\phi_{r}\left(x, s_{2}, \xi_{2}\right)\right| \leqq \omega(x, d, 3 r, \delta)
$$

or almost every $x \in \Omega$ and for $\left|s_{i}\right|<d,\left|\xi_{i}\right|<r(i=1,2)$, whenever $s_{1}-s_{2}|+| \xi_{1}-\xi_{2} \mid<\delta$. This inequality yields

$$
h_{u}(x)=\phi_{r}(x, u(x), D u(x))
$$

for every $u \in W^{1, \infty}\left(\Omega ; \mathbb{R}^{m}\right)$ with $\|D u\|_{L^{\infty}\left(\Omega ; \mathrm{R}^{n m}\right)}<r$ and for almost every $x \in \Omega$.

It remains to be proved that for all $(s, \xi)$ the function $x \mapsto \phi_{r}(x, s, \xi)$ is measurable. Let $s_{1} \in \mathbb{R}$ and let $u$ be affine with $u(0)=s_{1}, D u=\xi$. For almost every $x \in \Omega$ we have

$$
\phi_{r}\left(x, s_{1}+\xi \cdot x, \xi\right)=h_{u}(x)
$$



hence this function is measurable. If $\psi$ is a simple function, i.e. $\psi(x)=\sum_{i=1}^{k} s_{i} \chi_{E_{i}}(x)$,
with each $E_{i}$ measurable and $E_{i} \cap E_{j}=\emptyset$ if $i \neq j$, then

$$
\phi_{r}(x, \psi(x)+\xi \cdot x, \xi)=\sum_{i=1}^{k} \phi_{r}\left(x, s_{i}+\xi \cdot x, \xi\right) \chi_{E_{i}}(x) .
$$

Therefore by an approximation argument we can prove that $x \mapsto \phi_{r}(x, \theta(x)+$ $\xi \cdot x, \xi)$ is measurable, for $\theta \in L^{1}(\Omega)$. This happens in particular if $\theta(x)=s-$ $\xi \cdot x$, and the proof is complete.

The above lemma, together with the semicontinuity of $F(r, u, \Omega)$ and Remark [II.3], implies

Remark [II.4]. For every $(x, s, \xi) \in \Omega \times \mathbb{R}^{m} \times \mathbb{R}^{n m}$ set

$$
\phi(x, s, \xi)=\lim _{\substack{r \rightarrow \infty \\ r>|\xi|}} \phi_{r}(x, s, \xi)=\inf _{r>|\xi|} \phi_{r}(x, s, \xi) .
$$

The function $\phi$ is measurable in $x$, upper semi-continuous in $s$, continuous in $\xi$, and quasi-convex in $\xi$.

Let $\tilde{x} \in \Omega, \tilde{s} \in \mathbb{R}^{m}$. Lemma [III.3] implies that for all $r>0$ there exists a function $g_{r}^{(\tilde{x}, \tilde{s})}$ such that

Put

$$
\begin{gathered}
\int_{\Omega} g_{r}^{(\tilde{x}, \tilde{s})}(D u(x)) d x=\inf \left\{\liminf _{k \rightarrow \infty} \int_{\Omega} f\left(\tilde{x}, \tilde{s}, D u_{k}(x)\right) d x: u_{k} \rightarrow u\right. \\
\left.\left(\text { weak }^{*}\right) \text { in } W^{1, \infty}\left(\Omega ; \mathbb{R}^{m}\right) \text { and }\left\|D u_{k}\right\|_{L^{\infty}\left(\Omega ; \mathrm{R}^{n m}\right)} \leqq r\right\} \\
\text { for all } u \in W^{1, \infty}\left(\Omega ; \mathbb{R}^{m}\right) \text { with }\|D u\|_{L^{\infty}\left(\Omega ; \mathbb{R}^{n m}\right)}<r
\end{gathered}
$$

$$
g^{(\tilde{x}, \tilde{s})}(\xi)=\lim _{\substack{r \rightarrow \infty \\ r>|\xi|}} g_{r}^{(\tilde{x}, \tilde{s})}(\xi)
$$

Theorem [III.5]. For almost every $x \in \Omega$ and every $s \in \mathbb{R}^{m}$ the function $\xi \mapsto$ $\phi(x, s, \xi)$ is the greatest quasi-convex function less than or equal to $\xi \mapsto f(x, s, \xi)$.

Proof. Let $K \subset \Omega$ be a compact set such that $f$ is continuous on $K \times \mathbb{R}^{m} \times \mathbb{R}^{n m}$. For $x \in K, s \in \mathbb{R}^{m}$, set

$$
g_{r}(x, s, \xi)=g_{r}^{(x, s)}(\xi) .
$$

By the uniform continuity of $f$ on bounded subsets of $K \times \mathbb{R}^{m} \times \mathbb{R}^{n m}, g_{r}$ is continuous on $K \times \mathbb{R}^{m} \times\left\{\xi \in \mathbb{R}^{m m}:|\xi|<r\right\}$. Since $K$ is arbitrary, $g_{r}$ is defined for almost every $x \in \Omega$, and every $s \in \mathbb{R}^{m}, \xi \in \mathbb{R}^{n m}$, with $|\xi|<r$. Moreover, Lemma [I.5] implies that $g_{r}$ is a Carathéodory function, and it is quasi-convex in $\xi$ since the same holds for all $g_{r}^{(x, s)}$. As we remarked after Theorem [II.1], the functional

$$
G_{r}(u, \Omega)=\int_{\Omega} g_{r}(x, u(x), D u(x)) d x
$$

is sw*lsc on $\left\{u \in W^{1, \infty}\left(\Omega ; \mathbb{R}^{m}\right):\|D u\|_{L^{\infty}\left(\Omega ; \mathbf{R}^{n m}\right)}<r\right\}$. 
If we set, for all $x, s, \xi$,

$$
g(x, s, \xi)=g^{(x, s)}(\xi),
$$

then the functional

$$
u \mapsto \int_{\Omega} g(\tilde{x}, \tilde{s}, D u(x)) d x
$$

is the lsc envelope on $W^{1, \infty}\left(\Omega ; \mathbb{R}^{m}\right)$ of the functional

$$
u \mapsto \int_{\Omega} f(\tilde{x}, \tilde{s}, D u(x)) d x .
$$

Hence $\xi \mapsto g(\tilde{x}, \tilde{s}, \xi)$ is the greatest quasi-convex function not greater than $\xi \mapsto f(\tilde{x}, \bar{s}, \xi)$. This implies $g \geqq \phi$. For every $r>0, G_{r}$ is semicontinuous, hence $G_{r}(u, \Omega) \leqq F(r, u, \Omega)$, and $g_{r} \leqq \phi_{r}$, whence $g \leqq \phi$.

Note that the function $\phi$ does not necessarily represent the lsc envelope of $u \mapsto F(u, \Omega)$. Indeed, if $\phi$ is not a Carathéodory function, there is a counterexample even if $f$ is convex in $\xi$ (example 3.11 in [11]).

We give here some conditions which ensure that $\phi$ is a Carathéodory function.

Theorem [III.6]. If either of the conditions

(III.1) $f=f(x, \xi)$, or

(III.2) $\left|f\left(x, s_{1}, \xi\right)-f\left(x, s_{2}, \xi\right)\right|<\omega\left(x,\left|s_{1}-s_{2}\right|\right) \beta(|\xi|)$,

where $\omega: \mathbb{R}^{n} \times \mathbb{R} \rightarrow \mathbb{R}^{+}$is a Carathéodory function, $\omega(x, 0)=0$, and $\beta$ is increasing and non-negative,

is satisfied, then $\phi$ is a Carathéodory function.

Proof. If (III.1) holds, the result follows from Remark [III.4]. Next assume that (III.2) holds. We note (see [8], Corollary 2.4) that if $\psi: \mathbb{R}^{q} \rightarrow \mathbb{R}^{+}$is a convex function and if we set $M=\max _{|y| \leqq R} \psi(y)$, then for all $r<R$ and $y_{1}, y_{2} \in B_{r}(0)$ there holds

$$
\left|\psi\left(y_{1}\right)-\psi\left(y_{2}\right)\right| \leqq \frac{M}{R-r}\left|y_{1}-y_{2}\right|
$$

Since quasi-convexity implies weak quasi-convexity, this estimate shows that if $\psi: \mathbb{R}^{n m} \rightarrow \mathbb{R}^{+}$is quasi-convex then for all $r<R$ and $\xi_{1}, \xi_{2} \in B_{r}(0)$ we have

$$
\left|\psi\left(\xi_{1}\right)-\psi\left(\xi_{2}\right)\right| \leqq \frac{M \sqrt{\min (m, n)}}{R-r}\left|\xi_{1}-\xi_{2}\right|,
$$

where we have put $M=\max _{|\xi \leqq R|} \psi(\xi)$.

Note that $\phi$ almost everywhere satisfies the inequality

$$
\left|\phi\left(x, s_{1}, \xi\right)-\phi\left(x, s_{2}, \xi\right)\right|<\omega\left(x,\left|s_{1}-s_{2}\right|\right) \beta(|\xi|),
$$

as one can see by proving the same estimate for the function $g_{r}$ and then using the equality $g=\phi$. 
Choose $R>0$, and for all $x \in \Omega$ put

$$
\begin{aligned}
M(x) & =a(x)+\sup \{b(s, \xi):|s| \leqq R,|\xi| \leqq R\} \\
& \geqq \sup \{\phi(x, s, \xi):|s| \leqq R,|\xi| \leqq R\} .
\end{aligned}
$$

For almost all $x \in \Omega$ one has, for all $r<R, s_{1}, s_{2} \in B_{r}(0) \subset \mathbb{R}^{m}$, and $\xi_{1}, \xi_{2} \in$ $B_{r}(0) \subset \mathbb{R}^{n m}$,

$$
\begin{aligned}
\left|\phi\left(x, s_{1}, \xi_{1}\right)-\phi\left(x, s_{2}, \xi_{2}\right)\right| & \leqq \frac{M(x) \sqrt{\min (m, n)}}{R-r}\left|\xi_{1}-\xi_{2}\right|+ \\
& +\omega\left(x,\left|s_{1}-s_{2}\right|\right) \beta(R) .
\end{aligned}
$$

We summarize the results of section III as follows.

Statement [III.7]. Let $f: \mathbb{R}^{n} \times \mathbb{R}^{m} \times \mathbb{R}^{n m} \rightarrow \mathbb{R}$ be a Carathéodory function which satisfies (II.6) for some $p \geqq 1$ [or alternately satisfies (II.4)], and let either one of the conditions (III.1), (III.2) hold. Then the lsc envelope on $W^{1, p}\left(\Omega ; \mathbb{R}^{m}\right)$ [on $\left.W^{1, \infty}\left(\Omega ; \mathbb{R}^{m}\right)\right]$ of $u \mapsto F(u, \Omega)$ is the functional

$$
u \mapsto \int_{\Omega} \phi(x, u(x), D u(x)) d x,
$$

where for almost all $x \in \Omega$ and for all $s \in \mathbb{R}^{m}$ the function

$$
\xi \mapsto \phi(x, s, \xi)
$$

is the greatest quasi-convex function which is less than or equal to $\xi \mapsto f(x, s, \xi)$.

This theorem provides an extension of the results of [6] to the case in which $f$ depends on $u$ as well as on $x$ and $\xi$.

Note. This work was supported by the Italian government through the Consiglio Nazionale delle Ricerche.

\section{References}

1. Adams, R. A.: *Sobolev spaces, Academic Press, New York, 1975.

2. BALL, J. M.: On the calculus of variations and sequentially weakly continuous maps, Ordinary and partial differential equations (Proc. Fourth Conf., Univ. Dundee, Dundee 1976), pp. 13-25. Lecture Notes in Math., Vol.564, Springer, Berlin, Heidelberg, New York, 1976.

3. Ball, J. M.: Constitutive inequalities and existence theorems in nonlinear elastostatics, Nonlinear analysis and mechanics: Heriot-Watt Symposium (Edinburgh, 1976), Vol. I, pp. 187-241. Res. Notes in Math., No. 17, Pitman, London, 1977.

4. BaLl, J. M.: Convexity conditions and existence theorems in nonlinear elasticity, Arch. Rational Mech. Anal., Vol. 63 (1977), 337-403.

5. Ball, J. M.; Currie, J. C.; Olver, P. J.: Null lagrangians, weak continuity, and variational problems of any order, J. Funct. Anal., 41 (1981), 135-174.

6. Dacorogna, B.: A relaxation theorem and its application to the equilibrium of gases, Arch. Rational Mech. Anal., 77 (1981), 359-386. 
7. EISEN, G.: A selection lemma for sequences of measurable sets, and lower semicontinuity of multiple integrals, Manuscripta Math., 27 (1979), 73-79.

8. Ekeland, I.; Temam, R.: *Convex analysis and variational problems, Nortt Holland, Amsterdam, 1976.

9. Fusco, N.: Quasi-convessità e semicontinuità per integrali multipli di ordine superiore, Ricerche Mat., 29 (1980), 307-323.

10. LiU, F.-C.: A Luzin type property of Sobolev functions, Indiana Univ. Math. J., 26 (1977), 645-651.

11. Marcellini, P.; Sbordone, C.: Semicontinuity problems in the calculus of variations, Nonlinear Anal., 4 (1980), 241-257.

12. MeYers, N. G.: Quasi-convexity and lower semicontinuity of multiple variational integrals of any order. Trans. Amer. Math. Soc. 119 (1965), 125-149.

13. Morrey, C. B.: Quasi-convexity and the semicontinuity of multiple integrals. Pacific J. Math. 2 (1952), 25-53.

14. Morrey, C. B.: *Multiple integrals in the calculus of variations. Springer, Berlin, Heidelberg, New York 1966.

15. SERrin, J.: On the definition and properties of certain variational integrals. Trans. Amer. Math. Soc. 101 (1961), 139-167.

16. STEIN, E. M.: *Singular integrals and differentiability properties of functions. Princeton University Press, Princeton, 1970.

17. Tonelli, L.: La semicontinuità nel calcolo delle variazioni, Rend. Circ. Matem. Palermo 44 (1920), 167-249.

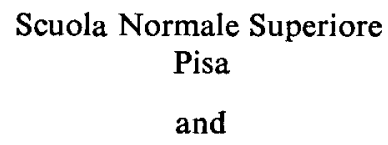

Istituto di Matematica "R. Caccioppoli"

Università di Napoli

(Received September 15, 1981) 